\title{
Patrones de distribución de los geométridos de la Región del Biobío, Chile: Una aproximación para su conservación
}

\author{
Distributional patterns of Geometridae of the Biobío Region, Chile: \\ An approach for their conservation
}

\author{
CARLOS ZAMORA-MANZUR ${ }^{1,2}$, LUIS E. PARRA ${ }^{2, ~}{ }^{*} \&$ EDILIA JAQUE ${ }^{3}$ \\ ${ }^{1}$ Departamento de Ecología, Facultad de Ciencias, Universidad Católica de la Santísima Concepción, \\ Alonso de Rivera 2850, Concepción, Chile \\ ${ }^{2}$ Departamento de Zoología, Facultad de Ciencias Naturales y Oceanográficas, Universidad de Concepción, \\ Casilla 160-C, Concepción, Chile \\ ${ }^{3}$ Departamento de Geografía, Facultad de Arquitectura, Urbanismo y Geografía, Universidad de Concepción, \\ Casilla 160-C, Concepción, Chile \\ *Autor correspondiente: luparra@udec.cl
}

\begin{abstract}
RESUMEN
La Región del Biobío en el centro sur de Chile es una zona de transición climática donde conviven bosques templados y esclerófilos, generando una zona de alta diversidad. Sin embargo, el área ha sido fuertemente afectada por la intervención antrópica y solo quedan algunos relictos de matorral y bosque nativo. Los geométridos, al igual que muchos insectos, están estrechamente asociados a la vegetación y por lo tanto serán afectados directamente por la intervención que se efectúe sobre esta. Así, en este estudio evaluamos los patrones de distribución y diversidad de geométridos en la Región del Biobío, con el objetivo de proponer sitios de alta prioridad para conservación mediante la identificación de áreas de endemismo y hot spots de diversidad. Los datos disponibles fueron procesados mediante análisis de parsimonia de endemismo y análisis de complementariedad, apoyados por SIG para rellenar vacíos y extrapolar la distribución de algunas especies de acuerdo a criterios establecidos. Encontramos una diversidad de 120 especies de geométridos, que corresponden a un $37.5 \%$ de la diversidad nacional, distribuidas en seis áreas de endemismo, localizadas en las formaciones vegetacionales más frecuentes en la Región del Biobío: bosque altomontano de Nahuelbuta, bosque esclerófilo de los arenales, bosque caducifolio de Concepción, bosque caducifolio andino del Biobío y bosque caducifolio de la frontera. El análisis de complementariedad determinó que 18 celdas contienen el número total de especies en la región. Basado en los análisis anteriores, se determinó que hay cinco áreas que deben ser consideradas como sitios de alta prioridad para la conservación de geométridos: (1) la ladera occidental de la Cordillera de Nahuelbuta y sector costero adyacente, (2) el área Pencopolitana de la zona litoral de la región, (3) Cerro Negro-Quillón, (4) Las Trancas, y (5) la zona del valle del Queuco-Alto Biobío.
\end{abstract}

Palabras clave: análisis de complementariedad, análisis de parsimonia de endemismos, conservación de insectos, endemismo, Lepidoptera.

\begin{abstract}
The Biobio Region in south-central Chile is a climatic transition area where temperate and sclerophyllous forests co-occur, generating a high diversity zone. However, the area has been strongly affected by antropic intervention and only a few relicts of native forest and shrubland are left. Geometrid moths, like many other insects, are closely associated to the vegetation and therefore they will be directly affected by antropic intervention. Thus, in this study we assessed the patterns of distribution and diversity of geometrids in the Biobio Region, aiming to propose high-priority sites for conservation by identifying endemism areas and diversity hot spots. The available data were processed by parsimony analysis of endemism and complementarity analysis, helped by GIS, for to fill up records and to extrapolate the distribution of some species according to established criteria. We found a diversity of 120 geometrid species, corresponding to $37.5 \%$ of the Chilean diversity, distributed in six endemism areas located in the most frequent vegetational formations in the Biobio region: the Nahuelbuta high-mountain forest, the sclerophyllous forest of sandy grounds, the deciduous forest of Concepción, the Andean deciduous forest of the Biobío, and the deciduous forest of the frontier. Complementarity analysis revealed that there were 18 square plots which included the total number of species in the region. Based on the above analyses, we determined that five areas should be considered as high-priority sites for the conservation of geometrids: (1) the western slope of the Nahuelbuta mountain range and its adjacent coastal sector, (2) the Pencopolitan area of the coastal zone of the region, (3) Cerro Negro-Quillón, (4) Las Trancas, and (5) the Alto Biobío-Queuco valley zone.
\end{abstract}

Key words: complementarity analysis, endemism, insect conservation, Lepidoptera, parsimony analysis of endemism. 


\section{INTRODUCCIÓN}

Los boques nativos australes de América del Sur constituyen una reserva mundial de biodiversidad, porque poseen una biota extraordinariamente rica en endemismos, particularmente géneros y familias monoespecíficas de plantas y animales (Armesto et al. 1996). Por ejemplo, el $80 \%$ de los anfibios, el $50 \%$ de los peces, el $36 \%$ de los reptiles, el $30 \%$ de las aves y el $33 \%$ de los mamíferos son endémicos. Gran parte de ellos presentan problemas serios de conservación, dado la alteración de sus hábitats originales por la acción antrópica (Glade 1993, Armesto et al. 1996). Los actuales parches de vegetación se generaron durante el último ciclo glacialinterglacial, los cuales sirvieron no solo como refugio glaciar sino también como reservorio y posterior centro de dispersión de especies (Villagrán 2001). Por la importancia de estos fenómenos en el patrón actual de distribución del bosque templado, la región de Chile central (entre $\operatorname{los} 29^{\circ}$ y los $40^{\circ} \mathrm{S}$ ) ha sido catalogada como uno de los hotspots de biodiversidad a nivel mundial, con 3429 especies vegetales y 335 especies de vertebrados (Myers et al. 2000). Sin embargo, estas son las regiones más afectadas por la intervención antrópica, así los bosques de la Cordillera de la Costa en las regiones VI, VII y VIII se encuentran prácticamente extintos y sus terrenos cubiertos por monocultivos de Pinus radiata, Eucalyptus globulus y E. nitens (Armesto et al. 1996, Grez et al. 2006).

El análisis del patrón de distribución latitudinal de la flora arbórea y del sotobosque de las comunidades forestales del sur de Chile, permiten establecer que los máximos niveles de riqueza de especies de todos los estratos se encuentran en un área geográficamente restringida, específicamente en la zona entre los ríos Maule y Valdivia (entre $36^{\circ}$ y $40^{\circ} \mathrm{S}$ ). Esta región concentra entre el 50 y $70 \%$ de las especies de plantas vasculares del bosque templado (Villagrán \& Armesto 2005).

La Región del Biobío se encuentra inserta en el desarrollo del bosque templado de Chile Central. Esta región se extiende entre los $36^{\circ} 00^{\prime}$ y $38^{\circ} 30^{\prime}$ de latitud Sur y desde los $71^{\circ} 00^{\prime}$ de longitud Oeste hasta el Océano Pacífico, abarcando una superficie de 37063 $\mathrm{km}^{2}$. Además, esta región se caracteriza por corresponder a un área de transición vegetacional, en donde se mezclan elementos de la región del matorral y del bosque esclerófilo de la zona central septentrional con la región del bosque caducifolio templado del sur de Chile (Gajardo 1994, Myers et al. 2000, Teneb et al. 2004). Desde una perspectiva evolutiva, las zonas de transición merecen especial atención, pues más que líneas estáticas representan áreas de interacción biótica intensa (Morrone 2004a). En esta zona los elementos florísticos principales o dominantes corresponden a formaciones dominadas por Acacia caven, Lithraea caustica y Quillaja saponaria, representantes del matorral esclerófilo (Gajardo 1994) (corotipos I y XIII según Teneb et al. 2004); Aextoxicon punctatum, Aristotelia chilensis y Nothofagus dombeyi, del bosque siempre verde (corotipos V, VI, X, XXII y XXIII según Teneb et al. 2004); Nothofagus antarctica, $N$. pumilio, N. glauca, N. alpina y Austrocedrus chilensis, representantes de la formación de bosque caducifolio (Gajardo, 1994) (corotipos XVI, XVII y XXI según Teneb et al. 2004). En la Región del Biobío existen 786 hectáreas de bosque nativo. Pero a pesar de la gran diversidad biológica que presenta, solo un $2.8 \%$ de la superficie están cubiertos por el Sistema Nacional de Áreas Protegidas del Estado (SNASPE).

La búsqueda de áreas de endemismos permite establecer áreas de importancia histórica con potencial para la propuesta de áreas de conservación (Escalante 2003a), y los insectos, por su diversidad, rapidez de crecimiento y la susceptibilidad al cambio ambiental, son excelentes bioindicadores, particularmente insectos fitófagos (e.g., Lepidoptera), los cuales probablemente presentan una especificidad a los taxa de plantas, existiendo congruencia entre la distribución de estos y sus hospederos (Holloway et al. 1987). Los lepidópteros, después de los coleópteros, corresponden al segundo orden más diversificado de los insectos. En la región Neotropical la familia Geometridae representa un 38 \% de la totalidad de especies, siendo solo superada por la familia Noctuidae (Heppner 1991). Para Chile se calcula la existencia de 451 especies de geométridos, de las cuales 277 son endémicas (Parra 1995) y todos sus hospederos conocidos son plantas nativas (Parra \& Beeche 1987, 
Parra \& Ibarra-Vidal 1996, Bocaz 2001, Bocaz et al. 2003).

Estudios anteriores realizados en geométridos de la región (Bocaz 2001, Pohl 2002, Bocaz et al. 2003, Bocaz \& Parra 2005) muestran patrones distribucionales y de asociación con comunidades vegetales a una escala local, que permite obtener buenos datos de diversidad y preferencias de hábitat de estos insectos. Sin embargo, es necesario proyectar estos estudios a toda la región con el fin de obtener mayor información sobre la diversidad regional y sus patrones distribucionales, que permitan discriminar áreas de alta riqueza específica y áreas de endemismo, ya sea a través de especies endémicas en sentido estricto o especies subendémicas (Rovito et al. 2004, Vergara et al. 2006), que puedan ser destinadas a la conservación de la biota regional y nacional. De esta forma y utilizando técnicas de biogeografía histórica y cuantitativa nos planteamos responder las siguientes interrogantes: ¿cuál es la diversidad taxonómica de geométridos en la Región del Biobío?; ¿la distribución de los geométridos está asociada a las formaciones vegetacionales descritas para la región, según Gajardo (1994)?; ¿están las especies de geométridos, descritas para la Región del Biobío, representadas en el sistema de áreas protegidas por el SNASPE?

Dado que los geométridos presentan una alta asociación a la vegetación nativa y que la Región del Biobío muestra altos niveles de diversidad vegetacional por encontrarse inmersa en una zona de transición climática, con una alta diversidad de macrounidades geomorfológicas regionales es esperable que la riqueza y diversidad de geométridos en esta región sea proporcionalmente alta al ser comparada con estos índices a nivel nacional y que la mayor riqueza de geométridos se encontraría en las zonas protegidas por el SNASPE y en aquellos sitios señalados como prioritarios para su conservación. El objetivo de este trabajo es conocer la diversidad de geométridos presentes en la Región del Biobío, delimitar rangos y determinar patrones de distribución de las especies descritas para la región y basado en ello determinar áreas de endemismo de geométridos para la región, que den pie para futuros proyectos de conservación.

\section{MÉTODOS}

\section{Área de estudio}

El área de estudio comprende toda la Región del Biobío, ubicada en la zona centro sur de Chile entre los $36^{\circ} 00^{\prime}$ y los $38^{\circ} 30^{\prime}$ S con una superficie de $37063 \mathrm{~km}^{2}$. La información sobre distribución de las especies de Geometridae se obtuvo de literatura, complementada con datos de la colección del Museo de Zoología de la Universidad de Concepción (UCCC-MZUC), Museo Nacional de Historia Natural (MNHN), Instituto de Entomología de la Universidad Metropolitana de Ciencias de la Educación (UMCE) y datos de muestreos provenientes de sectores de la región que presentaran un bajo número de registros. La localización de los sitios muestreados fueron georreferenciados en sistema de coordenadas geográficas, transformadas a coordenadas UTM (H18; ProvSAD '56), con lo que se generó una base de datos georreferenciada de los puntos muestreados y sus datos de especies asociados en el SIG Arc-View 3.2 (ESRI 2007).

Para la obtención de las Unidades Geográficas Operativas (OGUs) se utilizó una grilla con celdas de 0.25 grados de longitud por 0.25 grados de latitud que divide la región en 86 celdas, utilizada anteriormente por Jiménez (2002), Teneb et al. (2004) y Vergara et al. (2006) (Fig. 1A); asignando a cada celda las especies ubicadas en las localidades que interceptan (ver Apéndice). El tamaño de las celdas fue definido considerando que una grilla con celdas de mayor tamaño pierde poder de resolución, especialmente porque el área de estudio corresponde a un área pequeña fuertemente deteriorada por cultivos agrícolas y forestales (áreas naturales fragmentadas), Jiménez (2002); por lo tanto, una grilla con celdas de mayor tamaño no permitiría el reconocimiento de áreas de endemismo pequeñas o áreas de distribución disyuntas; mientras que celdas más pequeñas no permitirían reconocer unidades biogeográficas continuas (ver Casagranda et al. 2009).

La nomenclatura de especies arbóreas sigue a Rodríguez et al. (2005) y para especies de geométridos a Scoble (1999) en sus dos volúmenes.

\section{Extrapolación de distribución}

Debido que la región no ha sido muestreada a cabalidad se hizo necesario, para efectuar análisis posteriores, extrapolar la presencia de las diferentes especies de una celda hacia otras adyacentes que posean características similares para establecer un área potencialmente ocupada para cada especie. Para esto se realizó una superposición de las capas de información del uso de suelo (Conama-Conaf 1997), las formaciones vegetacionales de Gajardo (1994) y los sitios de muestreo, estableciendo un área buffer de $5 \mathrm{~km}$ de radio alrededor de cada punto de muestreo, siguiendo el siguiente procedimiento: (1) se seleccionaron los sitios de muestreo (puntos) y utilizándolos como centro se creó un círculo (buffer) de $5 \mathrm{~km}$ de radio alrededor de cada uno (simulando un radio de dispersión); (2) se seleccionaron solo los círculos que se prolongaban fuera de la celda original, hacia otra contigua. Si la celda contigua contenía al menos un $50 \%$ de la formación vegetacional correspondiente al sitio de muestreo y en ella se observa la presencia de parches de vegetación nativa, entonces se supuso que la especie se encontraba dentro de la celda. El buffer se estableció considerando que aunque la distancia que puede recorrer una polilla adulta podría llegar a cientos de kilómetros dependiendo de la especie y condiciones climáticas (Dantart et al. 2009), $5 \mathrm{~km}$ es una distancia muy conservadora y fácilmente alcanzable por la mayoría de las 


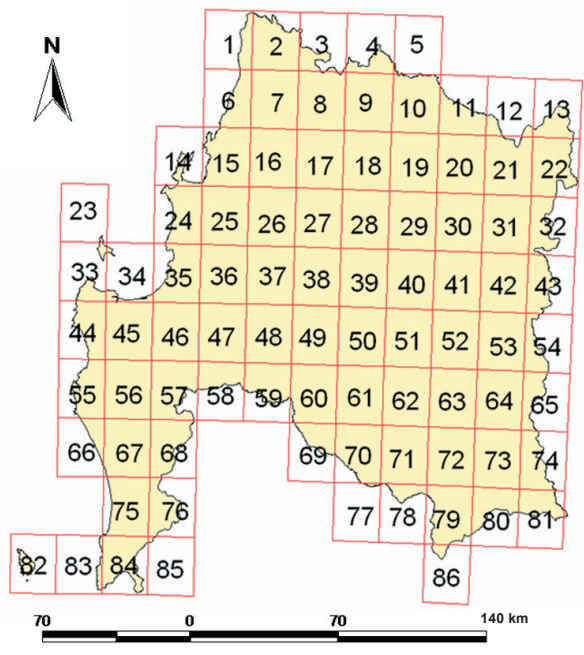

(A)
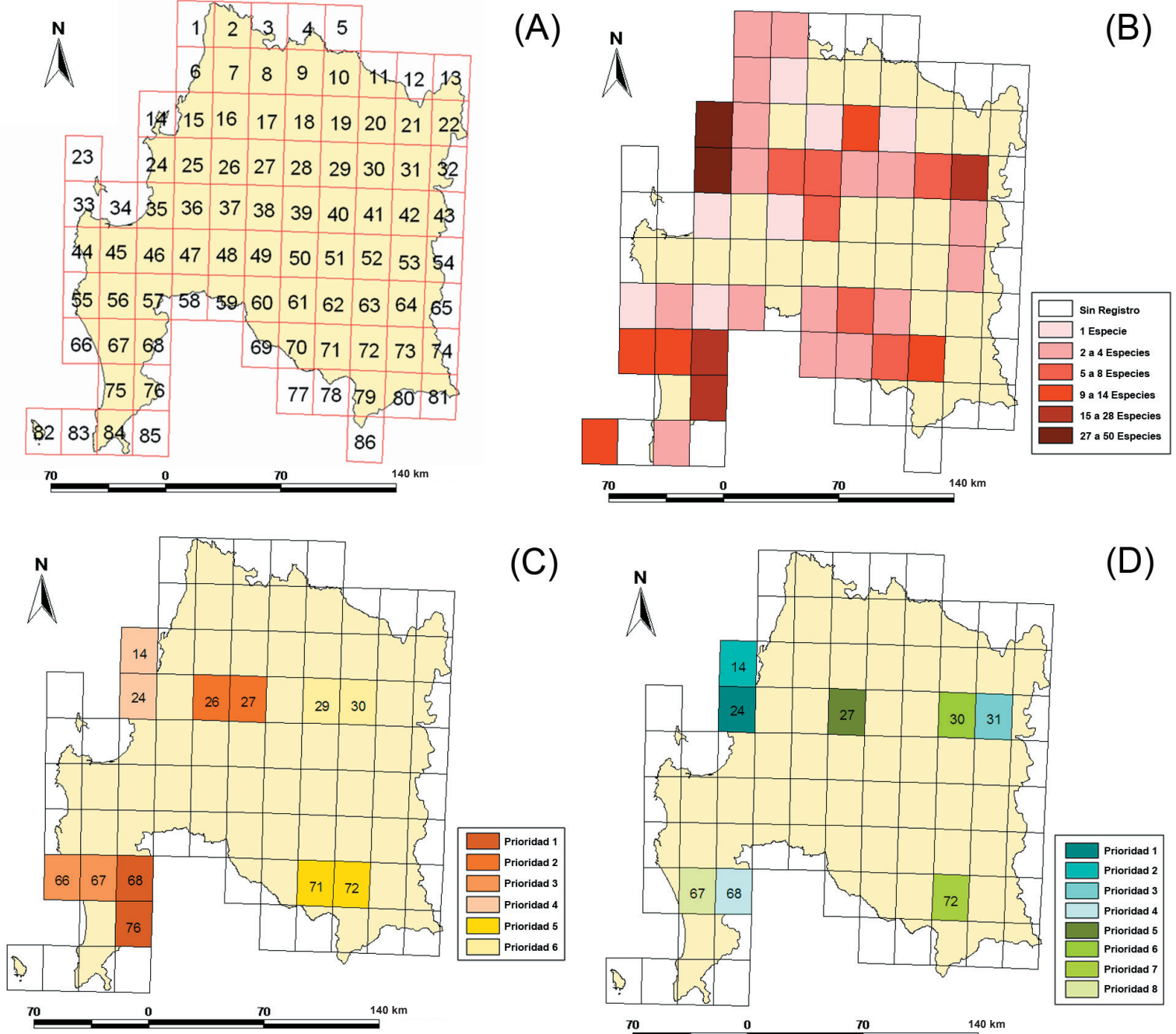

(C)
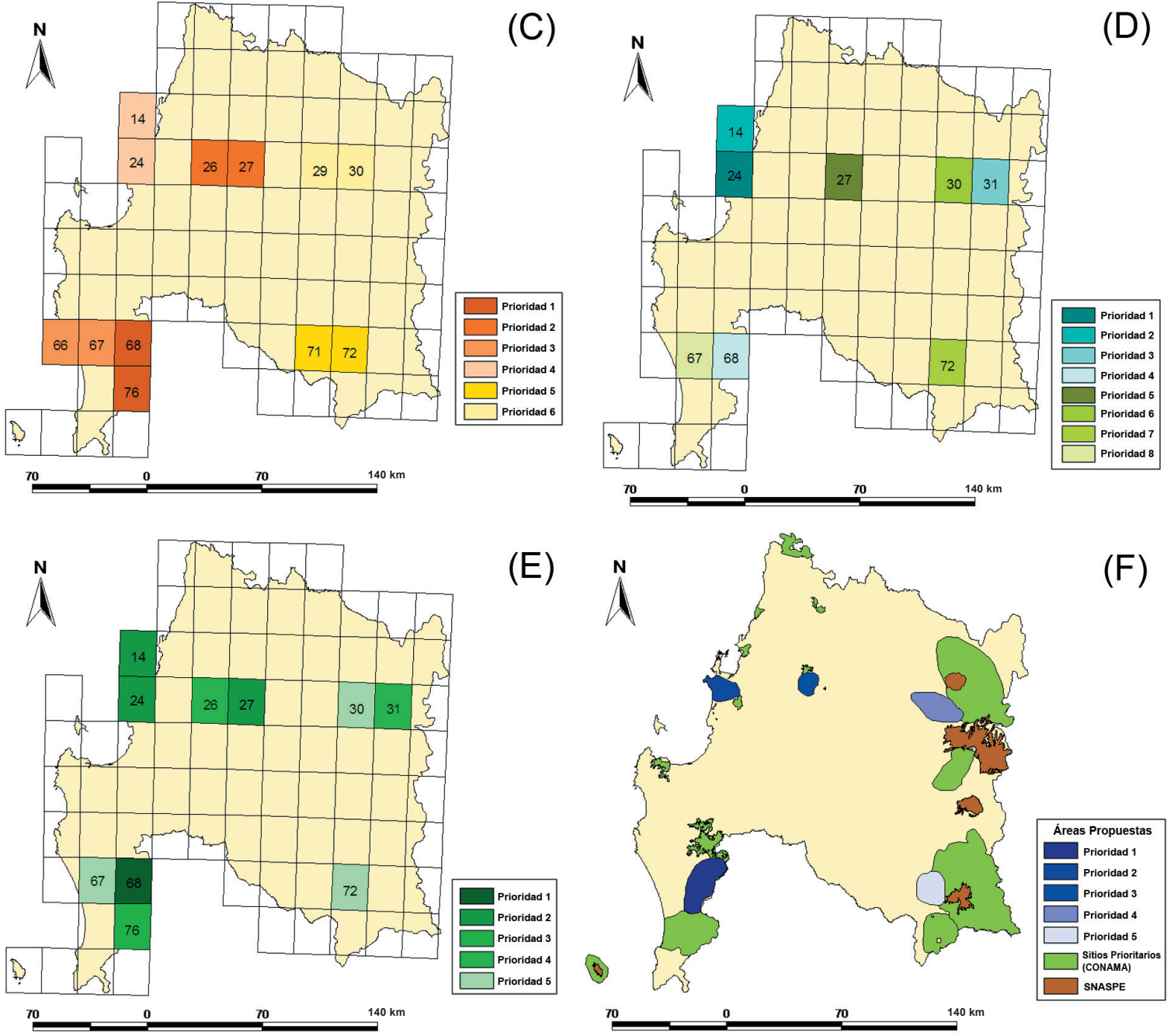
Fig. 1: (A) Mapa de la región dividido en 86 celdas (OGUs) de 0.25 grados de latitud y longitud. (B) Diversidad local (alfa) para cada celda, las tonalidades más oscuras representan una mayor diversidad de geométridos. (C) Áreas de endemismo propuestas por el Análisis de Parsimonia de Endemismos; las tonalidades naranja corresponden al PAE normal y los tonos amarillos al PAE con desconexión de ramas terminales, tonos más oscuros indican mayor prioridad de conservación. (D) Resultado gráfico del análisis de complementariedad con datos extrapolados; en tonos cian celdas con mayor prioridad, agrupando $82 \%$ de las especies totales y en tonos verdes las celdas con menor prioridad de conservación, en la leyenda se especifican las prioridades de conservación. (E) Resultado combinado del PAE y Análisis de Complementariedad, tonalidades más oscuras representan mayor prioridad de conservación. $(\mathrm{F})$ Resultado final y propuesta de sitios prioritarios; en azul las áreas propuestas por concentración de endemismos y riqueza de geométridos, tonalidades más oscuras indican mayor prioridad; en verde, Sitios Prioritarios propuestos por CONAMA; en castaño, áreas protegidas por el SNASPE.

(A) Map of the region divided into 86 squares (OGUs) of 0.25 degrees latitude and longitude. (B) Local diversity (alpha) for each grid, the darker colors represent a greater diversity of geometrid. (C) Area of endemism proposed by the Parsimony Analysis of Endemism, the orange tonalities correspond to a normal PAE and the yellow to a PAE with a disconnection of the terminal branches, darker colors indicate higher priority for conservation. (D) Graphical results of the analysis of complementary with extrapolated data; squares with a cyan tone indicates a higher priority, grouping $82 \%$ of total species, and in green tones the squares with a lower priority for conservation, the legend specifies conservation priorities. (E) Combined results of PAE and Complementarity Analysis, darker colors represent higher priority for conservation. (F) Final results and proposed priority sites; in blue the proposed areas by concentration of endemism and richness of geometrid, darker tones indicate higher priority; in green, priority sites proposed by CONAMA; brown areas are protected by SNASPE.

especies. Por otra parte, considerar un 50 \% de la formación vegetacional para asumir la presencia de la especie evita que ampliar la distribución hacia sectores muy intervenidos donde la presencia de la especie puede ser cuestionable.

Con las especies asignadas a las celdas de la grilla se obtuvo la diversidad de cada celda o diversidad alfa, diversidad alfa promedio, diversidad regional o gama y diversidad beta. Para calcular la diversidad beta se utilizó la formula de Whittaker (Murgía \& Rojas 2001). Para calcular la cantidad total de especies que debiera existir en la región se utilizó como estimador de riqueza el índice de Chao2 $\left(\mathrm{E}_{\mathrm{Ch}}\right)$ (Murgía \& Rojas 2001, Escalante 2003b). Una vez obtenidos los datos de distribución de cada especie asociada a las celdas de la grilla se creó una matriz de presencia y ausencia, la que fue utilizada para realizar los siguientes análisis: (1) Análisis de Parsimonia de Endemismos (PAE) (Morrone 1994) y (2) Análisis de Complementariedad (Vane-Wright et al. 1991).

\section{Análisis de parsimonia de endemismos (PAE)}

Se confeccionó una matriz de presencia/ausencia a la cual se le incorporó un área externa codificada con ceros para enraizar el cladograma (Morrone 2004b), se eliminaron las celdas donde no se obtuvo registros de especies como también las especies que generan autapomorfías, ya que estas no son relevantes para los resultados del análisis, el cual busca encontrar especies sinapomórficas para definir las áreas de endemismo. Para cada análisis independiente se estableció 1000 replicas, 100 árboles iniciales por replica, con la posibilidad de arrojar un máximo de 100000 árboles, con el procedimiento reiterativo de bisección y reconexión múltiple (Múltiple TBR + TBR). Posteriormente se buscó el árbol de consenso estricto. En ambos casos se calculó el largo o número de pasos, el Índice de Consistencia (Ci) y el Índice de Retención (Ri). Se delimitaron grupos de celdas definidos por al menos dos especies sinapomorficas, los cuales son catalogados como áreas de endemismo (Morrone 1994). Para estimar el valor de soporte de las ramas seleccionadas como áreas de endemismo se realizó un bootstrap con 1000 réplicas. Se realizó una desconexión de las especies que sustentaban las áreas de endemismo y se volvió a analizar la matriz (con las especificaciones ya descritas) para intentar descubrir nuevas áreas de concentración de endemismos que fuesen incompatibles con las obtenidas en el primer análisis (PAE-PCE: PAE with Progressive Character Elimination) (García-Barros et al. 2002, García-Barros 2003, Vergara et al. 2006). En forma paralela a la desconexión de especies, se desconectaron los terminales que fueron definidos en el primer análisis como áreas de endemismo y se analizó nuevamente la matriz, en busca de "áreas de endemismo secundarias". Se propone utilizar esta metodología en forma accesoria para definir nuevas áreas en función no solo de sus endemismos sino también de las especies que se distribuyen en alguna de las áreas propuestas por el primer análisis. Llamaremos a este análisis PAE con desconexión progresiva de terminales. Todos los análisis se llevaron a cabo mediante el programa de inferencia filogenética NONA 2.0 (Goloboff 1997) ejecutado desde el programa WinClada ver. 1.00.08 (Nixon 2002).

Las prioridades de conservación de las áreas encontradas se determinaron según la mayor cantidad de sinapomorfías y de ser estas iguales se consideró el número de especies totales. Se prefiere definir las prioridades solo con base en los endemismos, ya que el siguiente análisis (complementariedad) se basa en el número de especies totales.

\section{Análisis de complementariedad}

Este análisis permite identificar la diversidad biológica máxima en un número mínimo de áreas, lo que es un requisito primordial para la conservación (Vane-Wright et al. 1991, Scott 1997). Para establecer prioridades entre las celdas con base en la biodiversidad máxima, se empleó el complemento residual, que es el valor de la diferencia entre el número total de especies analizadas y el número de especies presentes en una celda (Faith 1994), por lo que la mayor diversidad biológica de un área específica es inversamente proporcional al valor del complemento residual. 
TABLA 1

Especies con amplia distribución en la Región del Biobío (en seis o más celdas) y números de la celdas en donde se encuentran.

Species with a wide distribution in the Biobío Region (in six or more cells) and numbers of the cells where they are.

\begin{tabular}{lcc}
\hline Taxones & $\mathrm{N}^{\text {o }}$ de registros & Celdas \\
\hline Catophoenissa dibapha & 6 & $1,14,18,24,25,71$ \\
Ennada flavaria & 7 & $14,15,18,24,67,72,82$ \\
Orthonama obstipata & 8 & $14,18,24,38,62,67,72,82$ \\
Syncirsodes primata & 9 & $14,24,25,27,35,38,67,71,82$ \\
Triptiloides fissa & 11 & $1,14,17,24,28,31,37,38,53,62,76$ \\
\hline
\end{tabular}

RESULTADOS

\section{Diversidad}

Para la Región del Biobío se obtuvieron 120 especies de geométridos repartidas en dos subfamilias y 61 géneros (Apéndice). Estas 120 especies representan un $37.5 \%$ de la diversidad nacional y los 61 géneros presentes en la región corresponden al $53 \%$ de los géneros citados para Chile. En la región se han realizado prospecciones en 60 puntos, sin embargo los datos utilizados en este estudio corresponden solo a 51 sitios de muestreo, ya que son los que poseen información suficiente para hacer posible su georreferenciación o al menos su correcta ubicación dentro de la grilla.

Antes de efectuar la extrapolación resulta evidente la presencia de algunas especies que, en comparación con la mayoría, tienen una distribución más homogénea en la región. Hay cinco especies que pueden considerarse de amplia distribución en la región (Tabla 1), que se encuentran distribuidas en al menos seis celdas.

La diversidad de cada una de las celdas (diversidad alfa), está resumida en la Fig. 1B. La diversidad promedio se estimó en 7.75 especies por celda, considerando solo las celdas con registros positivos. La diversidad alfa máxima está representada por la celda número 24 con un total de 50 especies.

Debido a que la región no ha podido ser muestreada en forma intensa y rigurosa, es posible distinguir un gran número de celdas sin registros y otras con solo un registro. Por ello, se aplicó el estimador no paramétrico de Chao2 (Murgía \& Rojas 2001, Escalante 2003b) para determinar la riqueza de especies esperada para la región, la cual se estimó en 179 especies. La Tabla 2 muestra los resultados de los análisis de diversidad, dispuestos en un cuadro resumen.

\section{Análisis de parsimonia de endemismos (PAE)}

Con el PAE es posible distinguir cuatro áreas de endemismos definidas por al menos dos especies no presentes en algún otro par de celdas (especies sinapomórficas). Las especies excluidas del análisis, por estar presentes solo en una celda, están listadas en el Apéndice. Se obtuvieron 560 árboles, con una longitud de 145 pasos (Ci: 47; Ri: 57), que generaron un árbol de consenso estricto de 178 pasos

\section{TABLA 2}

Cuadro resumen de los índices de diversidad de Geometridae para la Región del Biobío.

Summary table with the Geometridae diversity index for the Biobío Region.

\begin{tabular}{lc}
\hline Cualidad & Valores \\
\hline Subfamilias: & 2 \\
Géneros: & 61 \\
Diversidad gama: & 120 \\
Representatividad nacional: & $37.5 \%$ \\
Diversidad nacional: & 321 \\
Datos al extrapolar & \\
Diversidad alfa promedio: & 7.82 \\
Diversidad alfa máxima: & $50(\mathrm{OGU} \# 24)$ \\
Especies estimadas: & 179 \\
Diversidad beta: & 0.416 \\
\hline
\end{tabular}


(Ci: 38; Ri: 38). El árbol de consenso estricto (Fig. 2A) presenta altos niveles de homoplasia, lo que genera politomía. Sin embargo, en el mismo aparecen ramas sustentadas por dos o más especies sinapomórficas. Las áreas de endemismos corresponden a los clados formados por las celdas $26-27,66-67,14-24$ y 68 76. Las áreas propuestas, están sustentadas por: cuatro especies, en el caso de las celdas 26-27; tres especies para las celdas 66-67; seis especies para el clado $68-76$ y solo dos especies para la rama formada por los terminales 14 y 24 . Las especies que sustentan cada área se señalan en la Tabla 3.

(A)

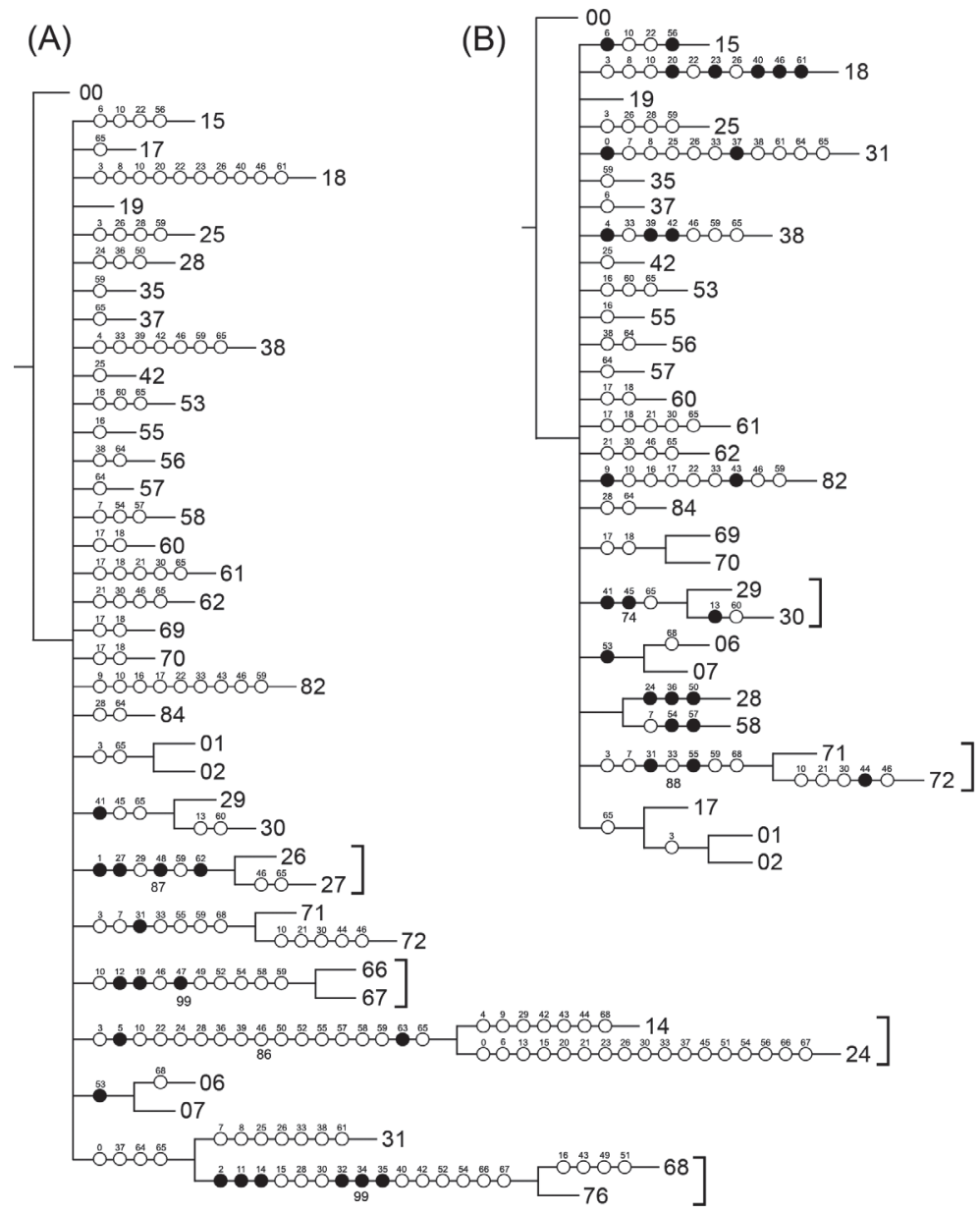

Fig. 2: Análisis de Parsimonia de Endemismos (PAE): (A) PAE sin desconexión de ramas; y (B) PAE con desconexión de ramas terminales. ] = áreas de endemismo; $\bullet=$ especies sinapomórficas; $\circ=$ especies homoplásicas. Los valores sobre las ramas corresponden al índice de bootstrap que sustentan las áreas de endemismos.

Parsimony Analysis of Endemism (PAE): (A) PAE without disconnection of the terminal branches; and (B) PAE with disconnection of the terminal branches. ] = areas of endemism; $\bullet=$ sinapomorphic species; $\circ=$ homoplasic species. The values on the branches correspond to the bootstrap support rate of endemic areas.
El índice de bootstrap arrojó valores entre 0.64 y 0.99 para las áreas de endemismos. Donde el mayor índice lo obtuvieron los clados 66-67 y 68-76 con un valor de 0.99 para cada uno. Las otras ramas formadas por el árbol de consenso estricto también reciben valores de bootstrap significativos.

\section{$P A E$ con eliminación de caracteres (PAE-PCE)}

La posterior aplicación del PAE-PCE, o PAE con eliminación progresiva de caracteres no generó nuevas áreas de endemismo debido a que no se alcanzó las dos sinapomorfías por rama (Morrone 1994) no calificando como áreas de endemismo.

B) 


\section{PAE con eliminación de ramas terminales}

Se procedió con la desconexión de ramas terminales completas, eliminando las cuatro áreas ya definidas. De esta forma el PAE arrojó 60 árboles con un largo de 75 pasos (Ci: 61; RI: 56), los cuales generaron un árbol de consenso estricto (Fig. 2B) de 97 pasos (Ci: 47; RI: 23) que presenta dos áreas de endemismo secundarias en las celdas 29-30 y 71-72. Se verificó la solidez de las ramas mediante un análisis del índice bootstrap. Las áreas 2930 y 71-72 fueron sustentadas por el índice con valores de 0.74 y 0.88 respectivamente; las especies que sustentan estas áreas se especifican en la Tabla 3 . El siguiente análisis, una vez eliminadas estas ramas terminales, no reveló nuevas áreas. La Fig. 1C muestra las áreas propuestas por el PAE y PAE con eliminación de ramas terminales, junto con las prioridades de conservación de cada área.

\section{Análisis de complementariedad}

Para el análisis de complementariedad, el menor valor del complemento residual (CR) corresponde a la celda $24(\mathrm{CR}=77)$, siendo esta la con mayor prioridad de conservar. El análisis determinó 16 celdas que juntas agrupan el total de especies de la región. Las celdas, ordenadas por prioridad de conservación son las siguientes: $24,14,31,68,27,30,72,67,61$, $18,82,38,56,42,6$ y 19 (Tabla 4 y Fig. 1D). Del listado anterior solo seis celdas fueron afectadas por la extrapolación, incorporando más especies a sus registros.

Considerando solo la congruencia entre las áreas propuestas por ambos análisis (PAE y complementariedad) se resuelven un total de 10 celdas con una alta prioridad de conservación. Estas 10 celdas se dividen en cinco prioridades de conservación agrupadas de la siguiente forma: (1) celda 68; (2) celdas 14,24 y 27; (3) celda 76; (4) celdas 26 y 31; (5) celdas 30,67 y 72; formando cinco áreas de concentración de especies (Fig. 1E).

Las especies excluidas del análisis (i.e. especies autapomórficas), aunque no fueron utilizadas para definir las áreas de conservación ni sus prioridades, es importante reconocer su estatus como "potenciales especies endémicas". Las celdas con mayor cantidad de especies autapomórficas corresponden a la 14 (13 spp.), la 24 (17 spp.) y la 31 (12 spp.). Las cuales ya forman parte de alguna de las áreas propuestas por los análisis anteriores.

Con estos resultados se generó la cartografía final, para delimitar con bordes más precisos las áreas que concentran la mayor cantidad de endemismos, subendemismos y riqueza de especies de geométridos en la Región del Biobío (Fig. 1F). Para ello se utilizó la sobreposición de cartografías de tipos forestales, curvas de nivel, registros de geométridos y registro por celda. A partir de esta información se delimitaron las cinco áreas provenientes del análisis PAE + Complementariedad: (1) vertiente occidental de la Cordillera de Nahuelbuta y sector costero aledaño; (2) área Pencopolitana de la zona litoral; (3) Cerro Negro - Quillón; (4) sector Las Trancas, Cordillera de Chillán en la Cordillera de los Andes; y (5) Alto Biobío y Valle del Queuco.

\section{TABLA 3}

Áreas de endemismos en orden de prioridad y especies (libres de homoplasia) que sustentan estas áreas.

Endemic areas in order of priority and species (free of homoplasy) that support these areas.

\begin{tabular}{|c|c|c|}
\hline Áreas & Celdas & Especies \\
\hline I & $68-76$ & $\begin{array}{l}\text { Catocalopsis medinae } \\
\text { Ennada pellicata } \\
\text { Eupithecia arauco } \\
\text { Lacaria schajovskoyi } \\
\text { Leucolithodes lecideata } \\
\text { Leucolithodes panteata }\end{array}$ \\
\hline II & $26-27$ & $\begin{array}{l}\text { Butleriana fumosa } \\
\text { Hoplosauris schausi } \\
\text { Perusia praecisaria } \\
\text { Thysanopyga conigera }\end{array}$ \\
\hline III & $66-67$ & $\begin{array}{l}\text { Euclidiodes meridionalis } \\
\text { Euangerona valdiviae } \\
\text { Perusia gracilis }\end{array}$ \\
\hline IV & $14-24$ & $\begin{array}{l}\text { Chlorotimandra viridis } \\
\text { Tomopteryx amoena }\end{array}$ \\
\hline $\mathrm{V}$ & $71-72$ & $\begin{array}{l}\text { Lacaria picuncharia } \\
\text { Rhinoligia biocellata }\end{array}$ \\
\hline VI & $29-30$ & $\begin{array}{l}\text { Mallomus penae } \\
\text { Oratha significata }\end{array}$ \\
\hline
\end{tabular}




\section{DISCUSIÓN}

La riqueza de geométridos en la Región del Biobío corresponde al $37.5 \%$ de las especies y $53 \%$ de los géneros citados para Chile. Estos altos niveles de diversidad se explican porque esta es una zona de transición vegetacional entre el Bosque Templado y Bosque Esclerófilo (Armesto et al. 1996), caracterizada por la gran diversidad de flora vascular (Villagran \& Armesto 2005). Esta genera una gran variedad de recursos y facilitaría la presencia de diversos representantes del gremio de defoliadores, como lo son los geométridos.

TABLA 4

Tabla resumen del Análisis de Complementariedad. Ranking $=$ Prioridad de Conservación; $\mathrm{N}^{\circ}$ celda $=$ número de celda; Contribución $=$ número de especies nuevas que aporta la selección de la celda en cuestión; Total = número de especies totales en la celda .

Summary of the Analysis of Complementarity. Ranking $=$ Conservation Priority, Cell $\mathrm{N}^{\circ}=$ cell number; Contribución $=$ number of new species that contributes the selection of the cell in question; Total = total number of species in the cell.

\begin{tabular}{lccc}
\hline Ranking & N $^{\text {o celda }}$ & Contribución & Total \\
\hline 1 & 24 & 50 & 50 \\
2 & 14 & 24 & 41 \\
3 & 31 & 18 & 23 \\
4 & $68^{*}$ & 12 & 26 \\
5 & $27^{*}$ & 4 & 8 \\
6 & $30^{\star}$ & 4 & 7 \\
7 & $72^{\star}$ & 3 & 14 \\
8 & 67 & 3 & 10 \\
9 & $61^{*}$ & 2 & 5 \\
10 & 18 & 1 & 11 \\
11 & 82 & 1 & 10 \\
12 & 38 & 1 & 8 \\
13 & 56 & 1 & 3 \\
14 & 42 & 1 & 2 \\
15 & $66^{*}$ & 1 & 2 \\
16 & 19 & 1 & 1 \\
\hline & & & \\
\hline
\end{tabular}

* celda extrapolada
Las áreas definidas con los análisis anteriores, cuatro áreas de endemismo y dos áreas de endemismo secundarias, se ubican en las formaciones vegetacionales de Gajardo (1994): (1) Bosque alto-montano de Nahuelbuta (celdas 68 y 76); (2) Bosque esclerófilo de los arenales (celdas 26 y 27); (3) Bosque caducifolio de Concepción (celdas 14-24 y 66-67); (4) Bosque caducifolio andino del Biobío (celdas 71 y 72); y (5) Bosque caducifolio de la frontera (celdas 29, 30 y 31). Todas estas formaciones vegetacionales originalmente tenían un gran desarrollo y riqueza florística en la región, pero ha sido completamente reemplazado por plantaciones forestales exóticas y cultivos agrícolas, encontrándose hoy en día restringidas a quebradas de difícil acceso. CONAMA (2006) ha establecido a muchas de estas zonas como sitios prioritarios de conservación. Estos sitios han sido propuestos por poseer una alta riqueza de especies vegetales y mantener características poblacionales y de intervención antrópica que hagan posible su conservación en el tiempo. La mayor concentración de especies de geométridos se observó en lugares vecinos a estos sitios propuestos por CONAMA como prioritarios para su conservación, lo que ratifica la importancia de estas zonas como reservorio de la biota nativa a nivel regional y nacional. Sin embargo, nuestros resultados no permiten hacer inferencias respecto a la presencia de geométridos en las áreas protegidas por el SNASPE, pero sí se verifica la presencia de especies endémicas en sectores aledaños a ellos.

A diferencia de los resultados aquí expresados, los coleópteros presentan índices de diversidad muy inferiores (Vergara et al. 2006). Probablemente, esto se debe a que en Coleoptera se trabajó en distintas categorías taxonómicas que implican distintos gremios funcionales, problema que se resolvería utilizando familias de coleópteros que presenten una especialización similar a la de Geometridae, es decir, que pertenezcan al gremio de los defoliadores y que harían más comparables nuestros resultados.

El análisis de parsimonia de endemismos es un método de biogeografía histórica que permite clasificar áreas o localidades de acuerdo a taxa compartidos (Morrone \& Crisci 1995). En esta línea, los análisis 
realizados, definen seis sectores como áreas de endemismo por presentar al menos dos especies compartidas en la rama interna de las áreas resueltas. Estas especies deben ser consideradas como endémicas al área en la cual se distribuyen dentro de la región, independientemente de ser o no endémicas a la Región del Biobío, por lo cual son considerados como taxa subendémicos (Rovito et al. 2004, Vergara et al. 2006).

La propuesta de PAE con desconexión de ramas terminales resultó ser una buena herramienta para la determinación de "áreas de endemismo secundarias". Las áreas de endemismo secundarias, además de contener sinapomorfías en sentido estricto, contienen especies que compartían con las áreas resueltas en un primer análisis. Estas áreas de endemismo secundarias cumplen el rol de proteger especies con una distribución mayor a las que sustentarían un PAE común.

La extrapolación resulta ser una buena herramienta; sin embargo, un aspecto importante a considerar al momento de extrapolar es el nivel de divergencia de los datos originales, intentando hacer una estimación más bien conservadora y relacionada a las características propias de los taxa estudiados como el grado de vagilidad, la asociación con la vegetación, necesidades climáticas, etc. Al comparar nuestros resultados, antes y después de la extrapolación fue posible identificar ciertas carencias que presentan los datos, como algunos sesgos hacia lugares de muestreo. Estos hacen que las estimaciones de diversidad regional alcancen valores muy elevados, que luego de la extrapolación descienden a niveles más reales. Este hecho demuestra que un esfuerzo de muestreo más uniforme, permitiría fortalecer los resultados de este tipo de estudios. En el PAE, las homoplasias también pueden ser consideradas evidencia de una falta de esfuerzo de muestreo entre dos zonas adyacentes (Navarro et al. 2007); y por otra parte pueden dar cuenta de áreas con conexión histórica las cuales pudieron sufrir procesos vicariantes tanto naturales como antrópicos (e.g., deforestación y reforestación con especies forestales introducidas) generando extinciones locales dentro de la región en estudio.

La degradación por cambio en el uso de suelo, destinados a asentamientos urbanos, cultivos agrícolas y plantaciones forestales, estaría explicando la gran cantidad de vacíos y baja diversidad en algunas celdas. La irregularidad en los datos de la diversidad local a nivel de celda, expresada en un valor de diversidad promedio baja, diversidad máxima muy alta y una cantidad considerable de celdas con una especie, se debe también a que las colectas no se han desarrollado en forma sistemática y que ciertos lugares geográficos han sido sobremuestreados en el tiempo, como ocurre en las cercanías de la ciudad de Concepción. La extrapolación realizada en este estudio viene a suplir este problema de muestreo, con el fin de obtener una clara visión de la distribución de las especies. En este sentido, resultaría de gran interés utilizar otros recursos o herramientas incorporadas en los SIG que permiten extrapolar condiciones de hábitat (en un sentido estrictamente espacial), disminuyendo así los errores que asocian la diversidad o presencia de especies en áreas definidas al interior de celdas geométricas (cuadrados) que no responden a patrones geográficos concretos.

En relación a las especies de amplia distribución, es posible predecir que su distribución no siga patrones en relación a la vegetación, por poseer hábitos polífagos o debido a que su planta hospedera presenta también una distribución amplia en la región. Teneb et al. (2004) hacen referencia a corotipos con especies vegetales de amplia distribución. Sería de gran ayuda en el futuro contar con trabajos destinados a conocer los hospederos de cada especie y de esta forma predecir la distribución de la especie con la de aquella planta y no necesariamente la distribución de una formación vegetacional completa, que solo corresponde a áreas potenciales de vegetación natural.

Las áreas definidas por el análisis de complementariedad corresponden básicamente a las mismas propuestas por el PAE, pero como este último se basa en las especies compartidas por un conjunto de áreas (sinapomorfías), no consideró en sus resultados la celda 31, que por el contrario, el análisis de complementariedad selecciona como una de las celdas con mayor prioridad. Otra diferencia con el PAE son las prioridades de conservación, que en el caso de este se consideró la cantidad de sinapomorfías sin hacer referencia a las especies totales. 
Debido a estas diferencias se realizó la unión de ambos análisis para entregar un resultado más robusto, eliminando las deficiencias de un análisis contrarrestadas por las fortalezas del otro. Las nuevas áreas y sus prioridades (Fig. 1F) son definidas tanto por el número de especies endémicas o subendémicas, como por una alta riqueza específica a nivel local.

Nuestras cinco propuestas de conservación se encuentran cerca o formando parte de los Sitios Prioritarios propuestos por CONAMA. Así, la ladera occidental de la Cordillera de Nahuelbuta y sector costero aledaño (1) se encuentra ubicado entre los Sitios Prioritarios Quebrada Caramavida y ADI Lleu-Lleu; el área Pencopolitana de la zona litoral (2) se encuentra agrupando el Santuario de la Naturaleza Península de Hualpén con el borde del Fundo Nonguén, catalogado como sitio prioritario; el Cerro Negro en Quillón (3) se encuentra cerca del sitio prioritario Cerro Cayumanque; el sector Las Trancas, cordillera de Chillán en la Cordillera de los Andes (4) está ubicado en el borde occidental del sitio prioritario Nevados de Chillán; Alto Biobío y el valle del Queuco (5) están ubicados al comienzo del sitio prioritario ADI Alto del Biobío. Los sitios prioritarios ADI Leu-Lleu y ADI Alto Biobío están propuestos con base en asentamientos indígenas, sin embargo, son áreas que presentan una gran cantidad de vegetación nativa al igual que los otros sitios propuestos por CONAMA, lo que determinaría la presencia de geométridos. Debido a que las propuestas de conservación para geométridos se encuentran muy cerca de los sitios prioritarios oficiales, es posible afirmar que los actuales sitios prioritarios para la conservación de la biodiversidad son una buena reserva de geométridos para la Región del Biobío.

Finalmente, una protección adecuada de la diversidad de geométridos en esta región debiera incluir como principales áreas a proteger los sitios prioritarios ya definidos por la CONAMA y adicionalmente sectores naturales cercanos al área urbana de Concepción (e.g., Península de Hualpén y Fundo Nonguén).

AGRADECIMIENTOS: Este estudio fue posible gracias al apoyo económico de la Dirección de Investigación de la Universidad de Concepción, a través de los proyectos 205.113.069-1.0 e Instrumental Científico 2001. Agradecemos también los asertivos comentarios de los revisores anónimos y del Dr. Patricio A. Camus para mejorar el presente manuscrito.

\section{LITERATURA CITADA}

ARMESTO J, P LEÓN-LOBOS \& MK ARROYO (1996) Los bosques templados del sur de Chile y Argentina: Una isla biogeográfica. En: Armesto J, C Villagrán \& MK Arroyo (eds) Ecología de los bosques nativos de Chile: 23-28. Editorial Universitaria, Santiago, Chile.

BOCAZ P, LE PARRA \& PF VICTORIANO (2003) Larval morphological variation and its relation to host plants in Syncirsodes primata (Lepidoptera: Geometridae). Gayana 67: 39-44.

BOCAZ P (2001) Taxonomía e historia natural de los geométridos presentes en la Península de Hualpén (VIII Región). Tesis de Pregrado, Facultad de Ciencias Naturales y Oceanográficas, Universidad de Concepción, Concepción, Chile.

BOCAZ P \& LE PARRA (2005) Revisión y bionomía del género Syncirsodes Butler 1882 (Lepidoptera: Geometridae). Revista Chilena de Historia Natural 78: 89-111.

CASAGRANDA MD, S ROIG-JUÑENT \& C SZUMIK (2009) Endemismo a diferentes escalas espaciales: Un ejemplo con Carabidae (Coleoptera: Insecta) de América del Sur austral. Revista Chilena de Historia Natural 82: 17-42.

CONAMA (2006) VIII Región-RRNN-Introducción. Recursos naturales de la región. Comisión Nacional del Medio Ambiente. URL: http:// www.conama.cl/portal/1255/article-26184.html (accedido Julio 14, 2006).

CONAMA-CONAF (1997) Catastro y evaluación de recursos vegetacionales nativos de Chile. Carta uso de suelo escala 1:50.000, VIII Región. Santiago, Chile.

DANTART J, C STEFANESCU, A ÁVILA \& M ALARCÓN (2009) Long-distance wind-borne dispersal of the moth Cornifrons ulceratalis (Lepidoptera: Crambidae: Evergestinae) into the northern Mediterranean. European Journal of Entomology 106: 225-229.

ESCALANTE T (2003a) Determinación de prioridades en las áreas de conservación para los mamíferos terrestres de México, empleando criterios biogeográficos. Anales del Instituto de Biología, Universidad Nacional Autónoma de México, Serie Zoología 74: 211-237.

ESCALANTE T (2003b) ¿Cuántas especies hay? Los estimadores no paramétricos de Chao. Elementos 52: 53-56.

ESRI (1997) Software ArcView GIS 3.2. Published by the Environmental Systems Research Institute. Inc. Redlands, New York, USA.

FAITH DP (1994) Genetic diversity and taxonomic priorities for conservation. Biological Conservation 68: 69-74.

GAJARDO R (1994) La vegetación natural de Chile. Clasificación y distribución geográfica. Editorial Universitaria, Santiago, Chile.

GARCÍA-BARROS E, P GURREA, M LUCIÁÑEZ, J MARTÍN-CANO, M MUNGUIRA et al. (2002) Parsimony analysis of endemicity and its application to animal and plant distributions in the Ibero-Balearic region (western Mediterranean). Journal of Biogeography 29: 109-124.

GARCÍA-BARROS E (2003) Mariposas diurnas 
endémicas de la Región Paleártica Occidental: Patrones de distribución y su análisis mediante parsimonia (Lepidoptera, Papilionoidea). Graellsia 59: 233-258.

GLADE A (1993) Libro rojo de los vertebrados terrestres de Chile. CONAF. Segunda edición. Ministerio de Agricultura, Santiago, Chile.

GOLOBOFF P (1997) NONA version 2.0. Published by the author.

HEPPNER JB (1991) Faunal regions and the diversity of Lepidoptera. Tropical Lepidoptera 2: 1-85.

GREZ AA, JA SIMONETTI \& RO BUSTAMANTE (2006) Biodiversidad en ambientes fragmentados de Chile: Patrones y procesos a diferentes escalas. Programa interdisciplinario de estudios en biodiversidad (PIEB), Universidad de Chile. Editorial Universitaria, Santiago.

HOLLOWAY JD, JD BRADLEY \& DJ CARTER (1987) CIE Guides to insects of importance to man. 1. Lepidoptera. The Cambrian News Ltd., Aberystwyth, UK.

JIMÉNEZ A (2002) Sitios prioritarios para la conservación de la flora en la VIII Región del Biobío, Chile. Tesis de Pregrado, Facultad de Ciencias Naturales y Oceanográficas, Universidad de Concepción, Concepción, Chile.

MORRONE JJ (1994) On the Identification of areas of endemism. Systematic Zoology 43: 438-441.

MORRONE JJ (2004a) La zona de transición sudamericana: Caracterización y relevancia evolutiva. Acta Entomológica Chilena 28: 41-50.

MORRONE JJ (2004b) Panbiogeografía, componentes bióticos y zonas de transición. Revista Brasileira de Entomologia 48: 149-162.

MORRONE JJ \& JV CRISCI (1995) Historical biogeography: Introduction to methods. Annual Review of Ecology and Systematic 26: 373-401.

MURGUÍA M \& F ROJAS (2001) Biogeografía cuantitativa. En: Llorente J \& J Morrone (eds) Introducción a la biogeografía en Latinoamérica: Teorías, conceptos, métodos y aplicaciones: 3947. Universidad Nacional Autónoma de México, México, DF.

MYERS N, R MITTERMEIER, C MITTERMEIER, G FONSECA \& J KENT (2000) Biodiversity hotspots for conservation priorities. Nature 403: 853-858.

NAVARRO JC, J LIRIA, H PIÑANGO \& R BARRERA (2007) Biogeographic area relationships in Venezuela: A parsimony analysis of CulicidaePhytotelmata distribution in national parks. Zootaxa 1547: 1-19.

NIXON KC (2002) WinClada 1.00.08. Published by the author.
PARRA LE (1995) Lepidoptera. En: Simonetti JA, MTK Arroyo, AE Spotorno \& E Lozada (eds) Diversidad biológica de Chile: 269-279. CONICYT, Santiago.

POHL C (2002) Correlation between Geometridae and vegetation on the Hualpén peninsula in Chile. Leistungsnachweis fauna/flora, landscape ecology. Tesis de Pregrado, Facultad de Ciencias Naturales y Oceanográficas, Universidad de Concepción, Concepción, Chile.

RODRÍGUEZ R, E RUIZ \& JP ELISSETCHE (2005) Árboles en Chile. Editorial Universidad de Concepción, Concepción, Chile.

ROVITO SM, M ARROYO \& P PLISCOFF (2004) Distributional modelling and parsimony analysis of endemicity of Senecio in the Mediterraneantype climate area of Central Chile. Journal of Biogeography 31: 1623-1636.

SCOBLE JM (ed) (1999) Geometrid moths of the world: A catalogue (Lepidoptera, Geometridae). CSIRO Publishing, Collingwood.

SCOTT JM (1997) Gap analysis for biodiversity survey and maintenance. En: Reaka-Kudla ML, DE Wilson \& EO Wilson (eds) Biodiversity II. Understanding and protecting our biological resources: 321-340. National Academy Press, Washington DC

TENEB EA, LA CAVIERES, MJ PARRA \& A MARTICORENA (2004) Patrones geográficos y de distribución de árboles y arbustos en la zona de transición climática mediterráneo-templada de Chile. Revista Chilena de Historia Natural 77: 51-71.

VANE-WRIGHT R, C HUMPHRIES \& P WILLIAMS (1991) What to protect? Systematics and the agony of choice. Biological Conservation 55: 235254.

VERGARA O, V JEREZ \& LE PARRA (2006) Diversidad y patrones de distribución de coleópteros en la Región del Biobío, Chile: Una aproximación preliminar para la conservación de la diversidad. Revista Chilena de Historia Natural 79: 369-388.

VILLAGRÁN C (2001) Un modelo de la historia de la vegetación de la Cordillera de la Costa de Chile central-sur: La hipótesis glacial de Darwin. Revista Chilena de Historia Natural 74: 793-803.

VILLAGRÁN C \& JJ ARMESTO (2005) Fitogeografía histórica de la Cordillera de la Costa de Chile. En: Smith-Ramírez C, J Armesto \& C Valdovinos (eds) Biodiversidad y ecología de los bosques costeros de Chile: 99-116. Editorial Universitaria, Santiago, Chile.

Editor Asociado: Sylvain Faugeron

Recibido el 19 de marzo de 2009; aceptado el 30 de agosto de 2011 


\section{APENDICE}

TABLA A1

Lista de especies de geométridos y su distribución en la región, ordenados por familia y género. List of geometrids species and their distribution in the region, sorted by family and gender.

\begin{tabular}{|c|c|c|c|}
\hline Subfamilia & Género & Especies & Celdas \\
\hline \multirow[t]{36}{*}{ Ennominae } & Aconcagua & Aconcagua parva (Butler) & $24,30,76$ \\
\hline & Acrosemia & Acrosemia quietaria (Felder \& Rogenhofer) & 14 \\
\hline & Catocalopsis & Catocalopsis medinae (Bartlett-Calvert) & 76 \\
\hline & Catophoenissa & Catophoenissa dibapha (Felder \& Rogenhofer) & $1,14,18,24,25,71$ \\
\hline & & Catophoenissa fuenzalidae Ureta & 24 \\
\hline & Chloroclydon & Chloroclydon rinodaria (Felder \& Rogenhofer) & 14,38 \\
\hline & Coironalia & Coironalia denticulada (Butler) & 15,24 \\
\hline & Dagostina & Dagostinia fasciata Orfila \& Schajovskoy & 24 \\
\hline & Dentinalia & Dentinalia forsteri Heimlich & 18,31 \\
\hline & & Ennada pellicata (Felder \& Rogenhofer) & 68,76 \\
\hline & Euangerona & Euangerona valdiviae Butler & 67 \\
\hline & Euclidiodes & Euclidiodes agitata (Butler) & 14 \\
\hline & & Euclidiodes beechei Parra & 14 \\
\hline & & Euclidiodes meridionales (Wallengren) & 67 \\
\hline & & Euclidiodes ophiusina (Butler) & 24,30 \\
\hline & Eusarca & Eusarca valdiviana (Butler) & 56 \\
\hline & Franciscoia & Franciscoia morenoi Orfila \& Schajovskoy & $24,62,72$ \\
\hline & Hasodima & Hasodima bartletti Parra \& Pascual-Toca & $14,15,18,24,82$ \\
\hline & & Hasodima boreas (Butler) & 24 \\
\hline & & Hasodima elegans Butler & 18,24 \\
\hline & Lacaria & Lacaria aczeli Orfila \& Schajovskoy & 72 \\
\hline & & Lacaria orfilai Rindge & $24,62,68,72$ \\
\hline & & Lacaria picuncharia Orfila \& Schajovskoy & 71 \\
\hline & & Lacaria schajovskoyi (Sperry) & 68 \\
\hline & Leucolithodes & Leucolithodes lecideata (Felder \& Rogenhofer) & 76 \\
\hline & & Leucolithodes panteata (Felder \& Rogenhofer) & 76 \\
\hline & & Leucolithodes paulina (Ureta) & 14,28 \\
\hline & Lozogramma & Lozogramma butyrosa Butler & 14 \\
\hline & & Lozogramma ceres Butler & 24 \\
\hline & Maeandrogonaria & Maeandrogonaria valentina Butler & $24,31,68$ \\
\hline & Malleco & Malleco versicolor Rindge & 31,56 \\
\hline & Mallomus & Mallomus chilenaria (Felder \& Rogenhofer) & 38 \\
\hline & & Mallomus danielae Parra & 18 \\
\hline & & Mallomus felderi (Butler) & 24 \\
\hline & & Mallomus latus (Rindge) & $14,24,38$ \\
\hline & & Mallomus mutabilis (Rindge) & 18,68 \\
\hline
\end{tabular}


TABLA A1. Continuación

\begin{tabular}{|c|c|c|c|}
\hline Subfamilia & Género & Especies & Celdas \\
\hline & & Mallomus penae (Rindge) & 29,30 \\
\hline & & Mallomus unda (Rindge) & 19 \\
\hline & Martindoelloia & Martindoelloia juradoi Orfila \& Schajovskoy & $14,38,68$ \\
\hline & \multirow[t]{2}{*}{ Microclysia } & Microclysia pristopera (Prout) & 68 \\
\hline & & Microclysia reticulata Butler & 68 \\
\hline & Microsema & Microsema plagiata (Butler) & 24 \\
\hline & Neorumia & Neorumia gigantea Bartlett-Calvert & 14 \\
\hline & Nucara & Nucara recurva Rindge & 14,72 \\
\hline & Opissogonia & Opisogonia diffissata Felder \& Rogenhofer & 14 \\
\hline & Oratha & Oratha significata Walker & 24,28 \\
\hline & \multirow[t]{5}{*}{ Perusia } & Perusia aurantiacaria (Blanchard) & 14 \\
\hline & & Perusia gracilis (Bartlett-Calvert) & 67 \\
\hline & & Perusia inusta (Felder \& Rogenhofer) & 14 \\
\hline & & Perusia praecisaria Herrich-Schäffer & 27 \\
\hline & & Perusia rubripicta Butler & 67,68 \\
\hline & Poya & Poya unica (Rindge) & 31 \\
\hline & Praeantarctia & Praeantarctia indecisa Heimlich & 31 \\
\hline & Proteopharmacis & $\begin{array}{c}\text { Proteopharmacis valdiviata (Felder \& } \\
\text { Rogenhofer) }\end{array}$ & $14,24,28$ \\
\hline & \multirow[t]{8}{*}{ Psilaspilates } & Psilaspilates catillata (Felder \& Rogenhofer) & 24 \\
\hline & & Psilaspilates cautinaria Parra & 68 \\
\hline & & Psilaspilates ceres (Butler) & 24,68 \\
\hline & & Psilaspilates concepcionensis Parra & 24 \\
\hline & & Psilaspilates obscura Parra & 24 \\
\hline & & Psilaspilates signistriata (Butler) & $14,24,67,68,76$ \\
\hline & & Psilaspilates stygiana (Butler) & 7 \\
\hline & & Psilaspilates venata (Butler) & $24,58,67,68$ \\
\hline & Pucaraia & Pucaraia izquierdii (Bartlett-Calvert) & 14 \\
\hline & Rhinoligia & Rhinoligia biocellata (Felder \& Rogenhofer) & $14,24,71$ \\
\hline & Rumia & Rumia aurantiacaria Blanhard & 24 \\
\hline & Syllexis & Syllexis lucida Butler & $14,24,58$ \\
\hline & \multirow[t]{3}{*}{ Syncirsodes } & Syncirsodes distictaria (Mabille) & $14,24,67$ \\
\hline & & Syncirsodes hyadesi (Mabille) & 42 \\
\hline & & Syncirsodes primata (Walker) & $\begin{array}{c}14,24,25,27,35,38,67, \\
71,82\end{array}$ \\
\hline & \multirow[t]{2}{*}{ Talca } & Talca absconda (Heimlich) & 18,31 \\
\hline & & Talca incurva Rindge & 31 \\
\hline & \multirow[t]{2}{*}{ Thysanopyga } & Thysanopyga varians (Butler) & 24 \\
\hline & & Thysanopyga conigera (Butler) & 27 \\
\hline
\end{tabular}


TABLA A1. Continuación

\begin{tabular}{|c|c|c|c|}
\hline Subfamilia & Género & Especies & Celdas \\
\hline \multirow[t]{38}{*}{ Larentiinae } & Anticlea & Anticlea chillanensis (Butler) & 30 \\
\hline & Butleriana & Butleriana fumosa (Butler) & 27 \\
\hline & Chlorotimandra & Chlorotimandra viridis Butler & 14,24 \\
\hline & Daniela & Danielaparra viridis (Parra) & $31,58,71$ \\
\hline & Ennada & Ennada blanchardi Parra \& Alvear & 14,82, \\
\hline & & Ennada flavaria Blanchard & $14,15,18,24,67,72,82$ \\
\hline & Eupithecia & Eupithecia cabrasae Rindge & 31 \\
\hline & & Eupithecia concepcion Rindge & 24,76 \\
\hline & & Eupithecia curacautinae Rindge & 31 \\
\hline & & Eupithecia horismoides Rindge & $53,55,68,82$ \\
\hline & & Eupithecia malchoensis Rindge & 31 \\
\hline & & Eupithecia mallecoensis Rindge & 61,82 \\
\hline & & Eupithecia oenone Butler & 14 \\
\hline & & Eupithecia petrohue Rindge & 31 \\
\hline & & Eupithecia picada Rindge & 31 \\
\hline & & Eupithecia recintoensis Rindge & 31 \\
\hline & & Eupithecia trancasae Rindge & 31 \\
\hline & & Eupithecia vallenarensis Rindge & 61 \\
\hline & & Eupithecia yelchoensis Rindge & 31 \\
\hline & Hagnagora & Hagnagora mesenata (Felder \& Rogenhofer) & 14 \\
\hline & Hoplosauris & Hoplosauris granitata (Fletcher) & 24 \\
\hline & & Hoplosauris heliconoides Butler & 30 \\
\hline & & Hoplosauris indistincta (Butler) & 24 \\
\hline & & Hoplosauris mabillei Parra & $14,24,27$ \\
\hline & & Hoplosauris macarenae Parra & 72 \\
\hline & & Hoplosauris pachrophylloides Parra & 31,42 \\
\hline & & Hoplosauris schausi (Warren) & $18,24,25,31$ \\
\hline & Isosauris & Isosauris cymatophora (Felder \& Rogenhofer) & 14,27 \\
\hline & Lagynopteryx & Lagynopteryx botulata (Felder \& Rogenhofer) & $24,31,38,71,82$ \\
\hline & Larentoides & Larentoides scarata (Felder \& Rogenhofer) & 24 \\
\hline & Nebula & Nebula diana (Butler) & $14,68,72$ \\
\hline & & Nebula mathewi (Butler) & 14 \\
\hline & Orthonama & Orthonama obstipata (Fabricius) & $\begin{array}{c}14,18,24,38,62,67 \\
72,82\end{array}$ \\
\hline & Pachrophylla & Pachrophylla linearia Blanchard & 24 \\
\hline & Parapachrophylla & Parapachrophylla claudiae Parra & 31 \\
\hline & Perizoma & Perizoma pastoralis (Butler) & 82 \\
\hline & Rindgenaria & Rindgenaria multilineata Parra & 24 \\
\hline & Rheumaptera & Rheumaptera exacta (Butler) & 15,24 \\
\hline
\end{tabular}


TABLA A1. Continuación

\begin{tabular}{cccc}
\hline Subfamilia & Género & Especies & Celdas \\
\hline Stamnodes & Stamnodes uniformata (Berg) & 30,53 \\
& Stamnodes sp. & 24 \\
Tomopteryx & Tomopteryx amoena Philippi & 14,24 \\
Triptila & Triptila virescens (Philippi) & $31,56,76,84$ \\
Triptiloides & Triptiloides fissa (Felder \& Rogenhofer) & $1,14,17,24,28,31,37$, \\
& & $38,53,62,76$ \\
& Triptiloides griseofasciata Parra & 24,76 \\
& Triptiloides krahmeri Parra \& Santos-Salas & 24,76 \\
& Triptiloides laeta (Philippi) & $6,14,71$ \\
& Xanthorhoe chiloena (Butler) & 14 \\
\hline
\end{tabular}

